heim). After the color reaction, the sections were counter stained with methyl green.

20. Tissues of pregnant mice were fixed in $10 \%$ buffered formalin and embedded in paraffin. Deparaffinized tissue sections were stained with hematoxylin and eosin, and other reagents such as periodic acidSchiff and Masson trichrome if necessary.

21. We thank the Laboratory Animal Research Center at the University of Tsukuba and T. Watanabe and T.
Mori for their technical advice and assistance. Care of experimental animals was within institutional guidelines. Supported by grants from the Ministry of Education, Science, Sports and Culture of Japan, and Circulation Biosystems at the University of Tsukuba. E.T. and J.I. are research fellows of the Japan Society for the Promotion of Science.

22 April 1996; accepted 5 September 1996

\title{
Laser Capture Microdissection
}

\author{
Michael R. Emmert-Buck, Robert F. Bonner, Paul D. Smith, \\ Rodrigo F. Chuaqui, Zhengping Zhuang, Seth R. Goldstein, \\ Rhonda A. Weiss, Lance A. Liotta*
}

\begin{abstract}
Laser capture microdissection (LCM) under direct microscopic visualization permits rapid one-step procurement of selected human cell populations from a section of complex, heterogeneous tissue. In this technique, a transparent thermoplastic film (ethylene vinyl acetate polymer) is applied to the surface of the tissue section on a standard glass histopathology slide; a carbon dioxide laser pulse then specifically activates the film above the cells of interest. Strong focal adhesion allows selective procurement of the targeted cells. Multiple examples of LCM transfer and tissue analysis, including polymerase chain reaction amplification of DNA and RNA, and enzyme recovery from transferred tissue are demonstrated.
\end{abstract}

As basic research links more and more genes to the cause of, predisposition for, or clinical behavior of specific diseases, the medical diagnostic laboratory will be transformed. Clinical molecular diagnostic testing will involve the measurement of simultaneous fluctuations of expression of multiple genes or genetic alterations occurring in developing or diseased tissues; thus, the next generation of molecular analysis methods will need to be miniaturized and automated. Several groups have proposed the development of image chips or array systems containing thousands of sequences for automated hybridization $(1,2)$. Each chip can simultaneously assay for several possible genetic mutations or measure the relative expression of multiple mRNA species (3). Alternatively, a serial analysis of gene expression (SAGE) approach can be used to simultaneously assess mRNA expression of multiple transcripts (4). With the use of polymerase chain reaction (PCR) amplification and such automated technology, molecular diagnostic testing in the future will likely consist of panels of tests rather than a few single tests for specific genes. In this way, a specific genetic fingerprint will be established for each individual lesion,

M. R. Emmert-Buck, R. F. Chuaqui, Z. Zhuang, R. A Weiss, L. A. Liotta, Laboratory of Pathology, National Cancer Institute, Room 2A33, Building 10, 9000 Rockville Pike, Bethesda, MD 20892, USA.

R. F. Bonner, P. D. Smith, S. R. Goldstein, Biomedical Engineering and Instrumentation Program, National Center for Research Resources, National Institutes of Health Bethesda, MD 20892, USA

*To whom correspondence should be addressed. which will be useful for diagnosis and prognosis and as a guide to therapy.

However, even the most sophisticated genetic testing methods will be of limited value if the input DNA, RNA, or proteins are not derived from pure populations of cells exhibiting the characteristic disease morphology. Several methods have been reported for tissue microdissection to address the problems associated with analysis of heterogeneous tissue. These include gross dissection of frozen tissue blocks to enrich for specific cell populations $(5,6)$, irradiation of manually ink-stained sections to destroy unwanted genetic material (7), touch preparations of frozen tissue specimens (8), and microdissection with manual tools (9-12). However, none of these methods offers the ease, precision, and efficiency necessary for routine research or clinical molecular diagnostic applications. For example, although manual microdissection can achieve good precision, it is time-consuming, labor-intensive, and requires a high degree of manual dexterity.

To overcome the drawbacks of current tissue microdissection techniques, we have developed a laser capture microdissection (LCM) system (Fig. 1). The method entails placing a thin transparent film over a tissue section, visualizing the tissue microscopically, and selectively adhering the cells of interest to the film with a fixed-position, short-duration, focused pulse from an infrared laser. The film with the procured tissue is then removed from the section and placed directly into DNA, RNA, or enzyme buffer for processing.

LCM has several advantages over current tissue microdissection approaches: It is simple, requires no moving parts, involves no manual microdissection or manipulations, and enables one-step transfers. The transferred tissue on the film retains its original morphology, thereby allowing microscopic verification of the specificity of the captured material. LCM can be performed almost as quickly as photography of histologic tissue sections. The use of sterile, disposable transfer films minimizes potential contamination, which is particularly important for PCR-based analyses. The films can be activated with minimal energy; small, inexpensive low-power lasers $(<50$ $\mathrm{mW}$ ) that can be attached to standard microscopes are sufficient. LCM has the potential to fulfill the critical needs of basic 9 researchers and clinicians to perform tissue microdissection on a routine basis.

The selective transfer and recovery of human tissue samples by LCM is shown in $\geq$ Fig. $2(13,14)$. These images represent the transfer of kidney glomeruli, Alzheimer's plaques in brain, in situ breast carcinoma, $\amalg$ atypical ductal hyperplasia (ADH) of the $\widetilde{0}$ breast, prostatic intraepithelial neoplasia on (PIN), and lymphoid follicles. We have found no limitations on the types of tissue $\mathbb{\widetilde { \sigma }}$ samples that can be procured by LCM and

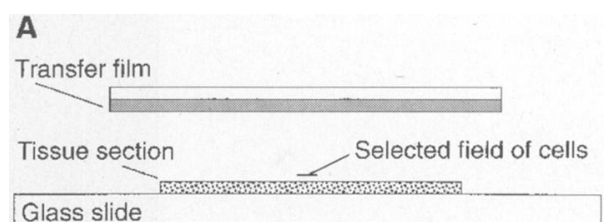

Glass slide

B

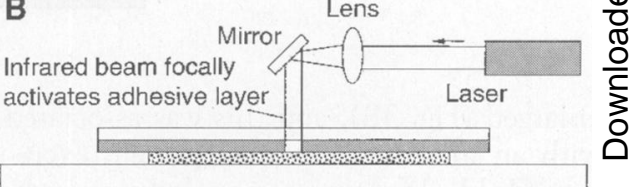

C

Transfer of selected cells

Fig. 1. (A) Transparent EVA thermoplastic film is applied to the surface of a routine tissue section mounted on a glass slide. The tissue-EVA film sandwich is viewed under a microscope, and the cells of interest are positioned in the center of the field. (B) A focused laser beam coaxial with the microscope optics is pulsed to activate the film, causing it to become focally adhesive and fuse to the selected underlying cells in the tissue section. (C) When the EVA film is removed from the tissue section, the selected cells remain adherent to the film surface. The film is then placed directly into the DNA, RNA, or enzyme buffer. The cellular material detaches from the film and is ready for standard processing. 
have performed transfers on a range of specimens, including frozen tissue samples, archival paraffin-embedded tissues (surgical and autopsy), and cytology cell preparations. Formalin- or alcohol-fixed tissues as well as hematoxylin and eosin-stained or unstained tissue can be transferred efficiently. Each laser pulse selectively transfers one small focal region of tissue or cell cluster to the film. Thus, a single region of tissue can be transferred to the film, or alternatively, the operator may move the slide and transfer multiple regions to the same film; for example, all the separate foci of a tumor or all the separate kidney glomeruli within a tissue section can be procured together on one film.

LCM is markedly faster and easier to perform than microdissection with manual tools. As an example, an individual glomerulus can be completely procured from a kidney tissue section in $<10 \mathrm{~s}$, and hun- dreds of glomeruli can be procured by an individual LCM operator in 1 hour with minimal effort. Manual methods cannot approach this level of microdissection in terms of speed or efficiency. Additionally, the LCM operator visualizes the transfer and can thus verify the specificity of the procured material. A potential disadvantage of manual microdissection is that fragments of pulverized tissue can detach from the tool and contaminate adjacent dissections. This difficulty is not encountered with LCM.

We observed little or no diminishment of PCR amplification of DNA or RNA after LCM. Additionally, native enzymes can be recovered and assayed for activity. Figure 3 illustrates examples of analysis after LCM transfer $(9,15-17)$. PCR amplification of DNA illustrates loss of heterozygosity (LOH) at several loci in tumors including the BRCAl gene in familial breast cancer, chromosome $8 \mathrm{p}$ in prostate cancer, the $p 16$ gene in invasive esophageal squamous cancer, and at the putative multiple endocrine neoplasia-1 (MEN1) tumor suppressor gene on chromosome 11q13 in a MEN1 gastrinoma (Fig. 3, B, D, E, F, and G). Other examples include single-strand conformation polymorphism (SSCP) detection of a single-base mutation in exon 2 of the Von Hippel-Lindau (VHL) gene in a hemangioblastoma (Fig. $3 \mathrm{H}$ ), reverse transcription (RT)-PCR amplification of actin and prostate-specific antigen (PSA) and recovery of active matrix metalloproteinase-2 (MMP2) from a frozen prostate cancer sample (Fig. 3, I, J, and L), and amplification of Mycobacterium tuberculosis DNA from a granulomatous lesion in the lung (Fig. 3K).

Transfer film activation can be achieved with a variety of lasers. Here, we used a carbon dioxide laser, which can be used in localized pulsed-laser microsurgery when target absorptivity is high $(18,19)$. Ther-
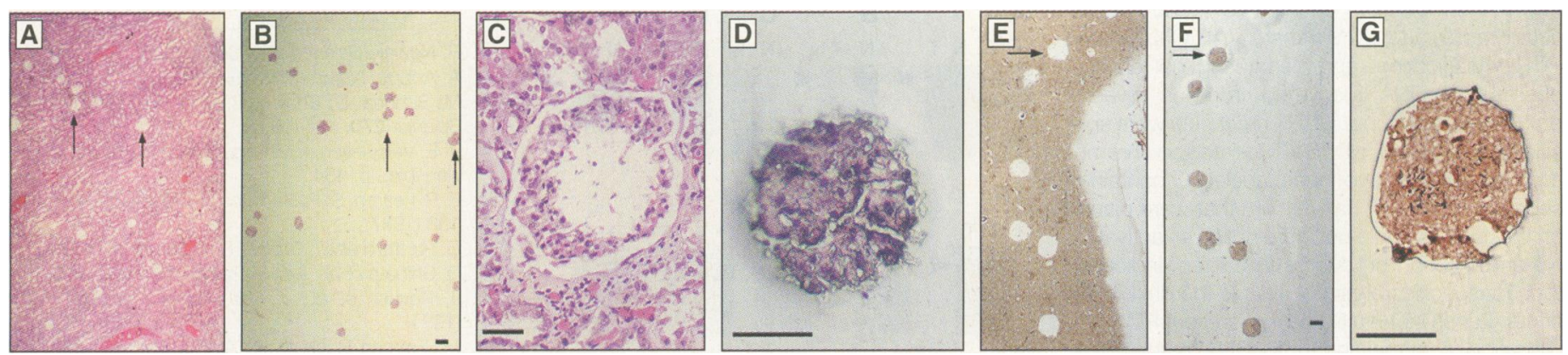

Fig. 2. Examples of LCM transfer. Sections were dehydrated, mounted on a glass slide, and stained with hematoxylin and eosin (H/E) or silver. (A to D) LCM transfer of kidney glomeruli. Several glomeruli were removed from the kidney cortex [(A), arrows] and were transferred to film [(B), H/E stain]; scale bar, $200 \mu \mathrm{m}$. The size and shape of the transfer region can be adjusted by altering the pulse characteristics of the laser and the number of laser pulses used to procure a lesion. The procured glomeruli in (B) vary in size from 60 to $150 \mu \mathrm{m}$. The same transfer is shown in (C) and (D) for a single glomerulus (scale bars, $50 \mu \mathrm{m}$ ); note the retained histology of the transferred tissue in (D). ( $\mathbf{E}$ to $\mathbf{G}$ ) LCM transfer of Alzheimer's plaques in brain. Multiple lesions were procured from a section of frontal cortex $[(E)$, arrow] and were transferred to film [(F), silver stain]; scale bar, $100 \mu \mathrm{m}$. A transferred lesion is shown in (G) (scale bar, $50 \mu \mathrm{m}$ ); note the darkly stained neurofibrillary tangle within the transferred tissue. $(\mathbf{H}$ and I) LCM transfer of in situ breast carcinoma, showing multiple regions of transfer. The lesion in the center of the field contains a region of central necrosis surrounded by viable neoplastic cells. LCM procurement (arrow) avoids both necrotic tissue and surrounding stroma (H/E stain; scale bar, 100
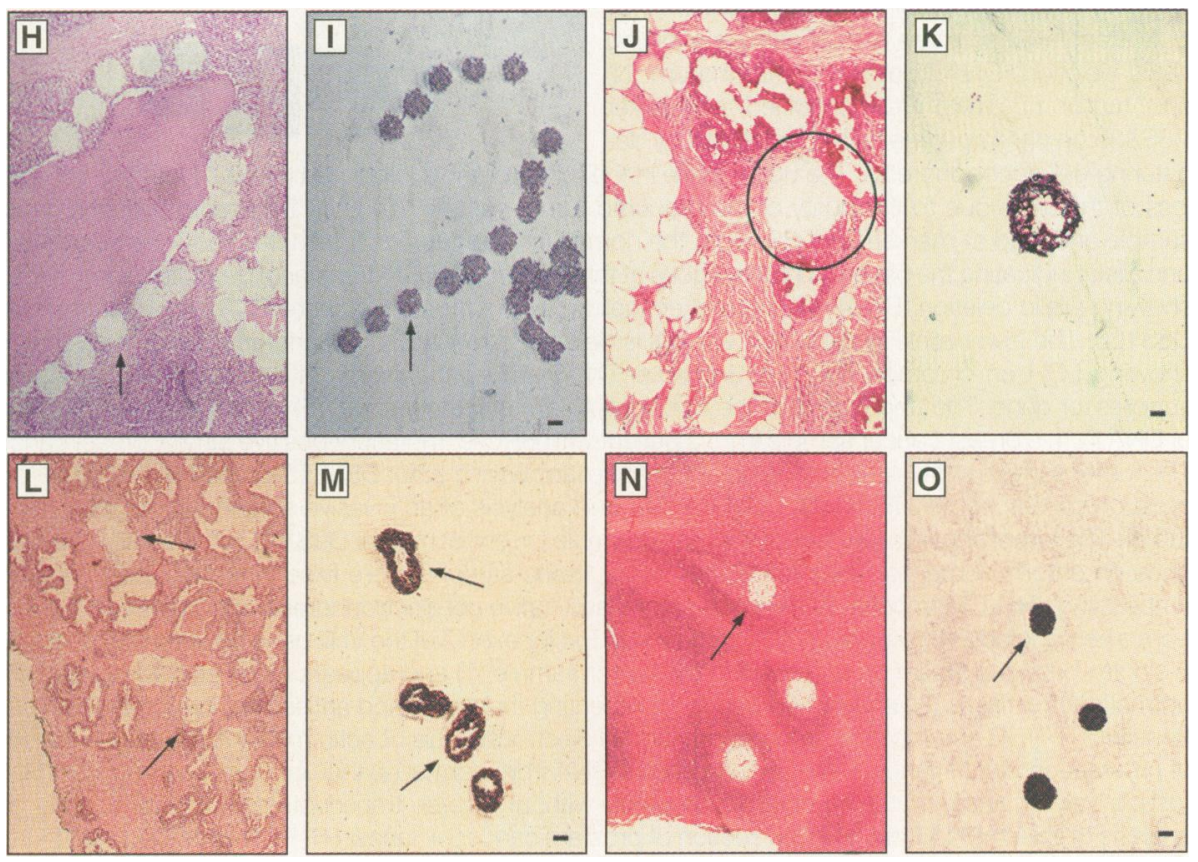

$\mu \mathrm{m})$. ( $\mathbf{J}$ and $\mathbf{K}) \mathrm{LCM}$ transfer of ADH of the breast.

$\mathrm{ADH}$ is the putative earliest precursor lesion of breast neoplasia and can occur several decades before the development of cancer. However, because of the small size of the lesion, little is known of the genetic alterations present in $A D H$. Note the incipient cribriforming pattern evident in the transferred lesion (circled area), a characteristic histopathological feature of $A D H(H / E$ stain; scale bar,
$100 \mu \mathrm{m}$ ). (L and $\mathbf{M})$ LCM transfer of PIN, a precursor lesion of prostate cancer. The transferred PIN foci are indicated by arrows (H/E stain; scale bar, 200 $\mu \mathrm{m})$. ( $\mathbf{N}$ and $\mathbf{O})$ LCM transfer of lymphoid germinal centers (arrows); the transferred regions are rich in $\mathrm{B}$ lymphocytes and are surrounded by the mantle zone, which was excluded (H/E stain; scale bar, $200 \mu \mathrm{m})$. 
moplastic films exhibit very strong absorption at carbon dioxide laser wavelengths, allowing localized heating of the 100- $\mu \mathrm{m}$ thick films with little direct absorption of the radiation by the underlying tissue. It is necessary for the targeted film volume to absorb sufficient energy to raise the film temperature to the fusion point and to provide its latent heat of fusion in the presence of lateral and axial heat conduction. The thermoplastic film then focally fuses to the surface of the adjacent tissue and cools within $200 \mathrm{~ms}$ of the end of the laser pulse. With this technique, the adherent tissue is exposed briefly to peak temperatures associated with the melting point of the film $\left(90^{\circ} \mathrm{C}\right)$. However, we have demonstrated that these brief thermal transients do not adversely affect our ability to perform subsequent PCR analysis or enzyme activity assays (Fig. 3).

In the field of oncology, the genetic analysis of microscopic premalignant lesions is important to basic researchers and has potential clinical implications (16, 20-23). These microscopic lesions, which represent an intermediate step of tumor progression from normal cells to frank cancer, can offer clues as to the nature of the fundamental alterations that underlie cancer development. Additionally, they represent potential markers of impending malignant transformation that could be used by clinicians to guide therapy or for heightened surveillance. LCM is ideal for microdissection of these small lesions (Fig. 2, H to M). Depending on the needs of the operator, the size of the tissue transfer can be varied by changing the diameter of the laser beam and pulse duration. We have demonstrated reproducible transfers in the 60 - to $700-\mu \mathrm{m}$-diameter range, primarily for procurement of small premalignant breast and prostate lesions (diameter 100

Fig. 3. Examples of PCR, RT-PCR, and enzyme activity assay after LCM transfer. (A) LCM transfer, PCR amplification, and denaturing gel electrophoresis of DNA recovered from an invasive breast cancer sample. PCR primer sets were specific for microsatellite D8S136 located on chromosome $8 \mathrm{p} 21$. The two lanes labeled $\mathrm{C}$ contained separate regions of transfer film that were placed on the tissue sample but were not laser-activated. No amplification product is visualized, indicating that nonspecific transfer of cells to the film did not occur. Lanes labeled $\mathrm{N}$ and T represent PCR amplification of normal and tumor DNA, respectively, showing the presence of two alleles (arrows). (B) LCM transfer of prostate cancer sample showing allelic deletion. DNA from normal epithelium $(N)$ and tumor (T) were PCR-amplified at marker D8S339 on chromosome 8p and analyzed by de-

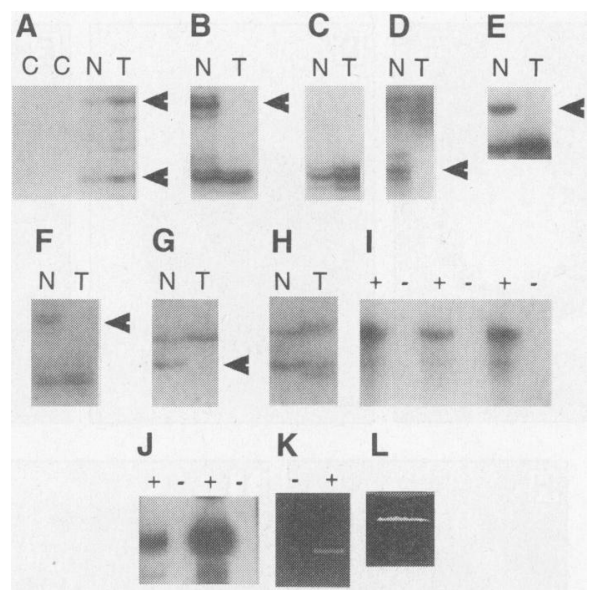
naturing gel electrophoresis. The upper allele in the tumor sample is deleted (arrow). Note the complete loss of the allele due to the purity of the procured tumor sample. (C) LCM transfer of prostate cancer sample analyzed at marker D8S339. Both the normal $(\mathrm{N})$ and tumor $(\mathrm{T})$ samples show the presence of one allele, indicating the patient is homozygous at this marker. (D) LCM transfer of prostate cancer sample showing allelic deletion. DNA from normal epithelium $(\mathrm{N})$ and tumor $(\mathrm{T})$ were PCR-amplified at marker D8S136. The lower allele in the tumor sample is deleted (arrow). (E) LCM and analysis of a gastrinoma showing LOH on chromosome 11q13 (marker D11S449) at the region of the putative MEN1 tumor suppressor gene. The upper allele in the tumor sample is deleted (arrow). (F) LCM transfer and analysis of DNA from a breast cancer sample from a patient with BRCA1 mutation-positive familial breast cancer. The tumor sample shows deletion of the upper allele (arrow) at marker D8S855 located within the BRCA1 gene on chromosome 17q21. (G) LCM transfer and analysis of an invasive squamous cell esophageal tumor. The tumor shows allelic loss in the tumor sample (arrow) at marker D9S171 in the vicinity of the p16 gene on chromosome 9p. The normal and tumor tissue samples were fixed in ethanol before paraffin embedding. (H) LCM transfer, PCR amplification, and native gel electrophoresis analysis (SSCP) of DNA from a hemangioblastoma, using PCR primers specific for exon 2 of the VHL gene on chromosome 3p25. A single-base pair mutation is present in the tumor sample ( $T$ ) and appears as an additional band. The normal control tissue ( $\mathrm{N}$ ) shows two bands representing the sense and antisense DNA strands. (I) LCM transfer, RT-PCR amplification, and denaturing gel electrophoresis of actin mRNA from a frozen section of prostate cancer (+ lanes, RT-PCR-amplified mRNA from normal epithelium, tumor, and control RNA, respectively; - lanes, matching controls prepared without reverse transcriptase). (J) LCM transfer and analysis of PSA mRNA from a frozen section of prostate cancer (+ lanes, RT-PCR-amplified mRNA from prostate tumor with $1 \times$ and $10 \times$ concentrations of template, respectively; - lanes, matching controls prepared without reverse transcriptase). (K) LCM transfer, PCR amplification, and gel electrophoresis of $M$. tuberculosis DNA from a granulomatous lesion in lung (- lane, DNA from normal lung adjacent to the lesion; + lane, DNA from a granuloma with histological documentation of acid-fast bacilli). (L) LCM transfer of frozen prostate cancer sample analyzed by gelatin zymography. Enzymatic clearing of the gel by MMP-2 (gelatinase A) results in the presence of a single band. $\mu \mathrm{m}$ to $1 \mathrm{~mm}$ ) without encroachment of adjacent nonneoplastic cells. Additionally, normal epithelium of the prostate or breast can be procured as matched control tissue by positioning the center of the laser within the lumen of a gland and using 10 to $20 \mu \mathrm{m}$ at the edge of the beam to selectively target the epithelium.

In most basic and clinical research studies, procurement of several hundred to several thousand cells is necessary to provide enough genetic material for reliable amplification and statistically meaningful analysis. Thus, transfers of tissue smaller than shown in Fig. 2 may not be needed for general diagnostic applications. However, because laser beams can be focused to a one-cell diameter $(\sim 10 \mu \mathrm{m})$, transfers of targeted single cells appear possible with this technique. The smallest transfer demonstrated in Fig. 2 is $60 \mu \mathrm{m}$, and the smallest transfers we have performed with the current system are $\sim 30 \mu \mathrm{m}$ (24).

\section{REFERENCES AND NOTES}

1. R. Nowak, Science 270, 368 (1995).

2. A. Abbott, Nature 379, 392 (1996).

3. M. Schena, D. Shalon, R. W. Davis, P. O. Brown Science 270, 467 (1995).

4. V. E. Velculescu, L. Zhang, B. Vogelstein, K. W. Kinzler, ibid., p. 484.

5. E. R. Fearon, S. R. Hamilton, B. Vogelstein, ibid. 238 193 (1987).

6. D. Radford et al., Cancer Res. 53, 2947 (1993).

7. D. Shibata et al., Am. J. Pathol. 141, 539 (1992).

8. J. Kovach et al., J. Natl. Cancer Inst. 83, 1004 (1991).

9. M. Emmert-Buck et al., Am. J. Pathol. 145, 1285 (1994).

10. Z. Zhuang et al., ibid. 146, 620 (1995).

11. S. Noguchi, K. Motomura, H. Inaji, S. Imaoka, $H$. Koyama, Cancer Res. 54, 1849 (1994).

12. T.-W. Park, J. C. Felix, T. C. Wright, ibid. 55, 4793 (1995).

13. Standard $6-\mu \mathrm{m}$ sections from formalin- or alcoholfixed, paraffin-embedded archival tissue samples were prepared on noncoated glass slides. Sections were deparaffinized, stained with hematoxylin and eosin, treated with 3\% glycerol in water for $1 \mathrm{~min}$ and air-dried for $5 \mathrm{~min}$ before LCM. Fresh tissue was snap-frozen immediately after surgery at $-70^{\circ} \mathrm{C}$. Cryostat sections $(6 \mu \mathrm{m})$ were prepared on standard glass histology slides. Tissue sections were fixed in formalin or alcohol and stained with hematoxylin and eosin (Lerner Laboratories, Pittsburgh, PA). Sections were dehydrated in graded alcohols and air-dried for 5 min before LCM transfer.

14. Flat films ( $100 \mu \mathrm{m}$ thick) were made by spreading a molten thermoplastic material lethylene vinyl acetate polymer (EVA); Adhesive Technologies, Hampton, $\mathrm{NH}$ ] on smooth silicone or polytetrafluoroethylene surfaces. The optically transparent thin films were placed on top of tissue sections, and the tissue-film sandwich was viewed in an inverted microscope (Olympus Model CK2, Tokyo) at 100× magnification (10× objective). A pulsed carbon dioxide laser beam was introduced by way of a small front-surface mirror coaxial with the condenser optical path so as to irradiate the upper surface of the EVA film. The carbon dioxide laser (either Apollo Company model 580, Los Angeles, or California Laser Company model LS150, San Marcos, CA) provided individua pulses of adjustable pulse length and power. A ZnSe lens focused the laser beam to an adjustable spot size on the target specimen. For transfer spots of $150 \mu \mathrm{m}$ diameter, a 600 -ms pulse delivered 25 to 30 
$\mathrm{mW}$ to the film. For smaller or larger spots, the power was decreased or increased approximately in proportion to the diameter of the laser spot focused on the tissue. The absorption coefficient of the EVA film measured by Fourier transform infrared spectroscopy as well as by direct transmission, was $200 \mathrm{~cm}^{-1}$ a the laser wavelength $(10.6 \mu \mathrm{m})$. Because $>90 \%$ of the laser radiation was absorbed within the thermoplastic film, little direct heating of the tissue specimen by the laser occurred. The glass slide provided a large heat sink that confined the full-thickness transient focal melting of the thermoplastic material to the targeted region. The focally molten plastic wet the targeted tissue. After cooling and recrystallization, the film formed a local surface bond to the targeted tissue that was stronger than the adhesion forces of the tissue to the slide. The film and targeted cells were removed from the tissue specimen, resulting in focal microtransfer of the targeted tissue to the film surface.

15. For PCR, the transfer film and adherent cells were immediately resuspended in $40 \mu \mathrm{l}$ of $10 \mathrm{mM}$ tris- $\mathrm{HCl}$ (pH 8.0), 1 mM EDTA, 1\% Tween-20, and proteinase $\mathrm{K}(0.1 \mathrm{mg} / \mathrm{ml})$ and incubated overnight at $37^{\circ} \mathrm{C}$. The mixture was boiled for $10 \mathrm{~min}$ to inactivate the proteinase K. The tubes were briefly spun (1000 rpm, 1 $\mathrm{min}$ ) to remove the film, and $0.5 \mu \mathrm{l}$ of the supernatant was used for PCR. For the most efficient tissue recovery, the transfer film was initially applied to the tissue section as a circular disk (diameter $0.5 \mathrm{~cm}$ ). After LCM transfer, the disk was placed into a well in a 96-well microtiter plate containing $40 \mu$ l of extraction buffer. The oligonucleotide primers for the polymorphic DNA markers were purchased from Research Genetics (Huntsville, AL). Loci were D8S136 and D8S339 located on chromosome 8p, D17S855 on chromosome 17q21, D11S449 on chromosome 11q13, D9S171 on chromosome 9p, specific primers for exon 2 of the $\mathrm{VHL}$ gene, and specific primers for $M$. tuberculosis. All PCR reactions used incorporation of $\left[{ }^{32} \mathrm{P}\right] d$ deoxycytidine triphosphate for visualization of product except amplification of $M$ tuberculosis, which was visualized by ethidium bromide staining.
16. M. Emmert-Buck et al., Cancer Res. 55, 2959 (1995).

17. For RT-PCR, total RNA was extracted from tissue after LCM by means of a modification of an RNA microisolation protocol (Stratagene). Volumes were proportionally adjusted downward, and a 10-fold increase in glycogen carrier $(10 \mathrm{ng} / \mathrm{ml})$ was used in all precipitation steps. After initial recovery and resuspension of the RNA pellet, a deoxyribonuclease (DNase) step was performed for 3 hours at $37^{\circ} \mathrm{C}$ using DNase (10 U/ml; GenHunter, Nashville, TN) in the presence of $4 \mathrm{U}$ of RNase Inhibitor (PerkinElmer), followed by reextraction of the RNA. The resuspended RNA was reverse-transcribed using 5 $\mu \mathrm{M}$ random hexamer primers (Perkin-Elmer), 250 $\mathrm{mM}$ deoxynucleotide triphosphate, and $100 \mathrm{U}$ of reverse transcriptase (MMLV, GenHunter). PCR was performed with specific actin or PSA primers, and the products were subjected to denaturing electrophoresis gel analysis

18. S. Meyers, R. F. Bonner, M. M. Rodrigues, E. J. Ballantine, Ophthalmology 90, 563 (1983).

19. J. Walsh, T. J. Flotte, R. R. Anderson, T. F. Deutsch, Lasers Surg. Med. 8, 108 (1988).

20. P. O'Connell, V. Pekkel, S. Fuqua, C. K. Osborne, D. C. Allred, Breast Cancer Res. Treat. 32, 5 (1994).

21. Z. Zhuang, M. J. Merino, R. F. Chuaqui, L. A. Liotta, M. R. Emmert-Buck, Cancer Res. 55, 467 (1995).

22. D. Page, W. D. Dupont, L. W. Rogers, M. S. Rados, Cancer 55, 2698 (1985).

23. J. McNeal and D. G. Bostwick, Hum. Pathol. 17, 64 (1986).

24. We are currently optimizing thinner, smoother transfer films and finer laser activation, which may allow for reproducible transfer of targeted single cells (R. F. Bonner et al., in preparation)

25. We thank S. K. Apple, J. P. Struewing, W. M. Linehan, C. D. Vocke, G. Oliver, A. Lash, M. J. Roth, M. J. Merino, P. H. Duray, I. A. Lubensky, L. V. Debelenko, V. E. Norman, and A. Coleman for tissue samples, technical support, or editing of the manuscript.

30 May 1996; accepted 16 September 1996

\section{Structure of the p53 Tumor Suppressor Bound to the Ankyrin and SH3 Domains of 53BP2}

\section{Svetlana Gorina and Nikola P. Pavletich}

Mutations in the p53 tumor suppressor are among the most frequently observed genetic alterations in human cancer and map to the 200-amino acid core domain of the protein. The core domain contains the sequence-specific DNA binding activity and the in vitro 53BP2 protein binding activity of p53. The crystal structure of the p53 core domain bound to the 53BP2 protein, which contains an SH3 (Src homology 3) domain and four ankyrin repeats, revealed that (i) the SH3 domain binds the L3 loop of p53 in a manner distinct from that of previously characterized SH3-polyproline peptide complexes, and (ii) an ankyrin repeat, which forms an L-shaped structure consisting of a $\beta$ hairpin and two $\alpha$ helices, binds the L2 loop of p53. The structure of the complex shows that the 53BP2 binding site on the p53 core domain consists of evolutionarily conserved regions that are frequently mutated in cancer and that it overlaps the site of DNA binding. The six most frequently observed p53 mutations disrupt 53BP2 binding in vitro. The structure provides evidence that the 53BP2-p53 complex forms in vivo and may have a critical role in the p53 pathway of tumor suppression.

The p53 tumor suppressor pathway $(1,2)$ has a central role in suppressing neoplastic transformation as it can respond to DNA damage by inducing cell cycle arrest (3) or

Cellular Biochemistry and Biophysics Program, Memorial Sloan-Kettering Cancer Center, New York, NY 10021, USA apoptosis $(4,5)$. The cell cycle arrest is mediated, at least in part, by p53's sequence-specific DNA binding (6) and transactivation functions (7), which can induce the expression of the p21(Cip1WAF1) cyclin-dependent kinase inhibitor, arresting the cell cycle (8). However, p21-

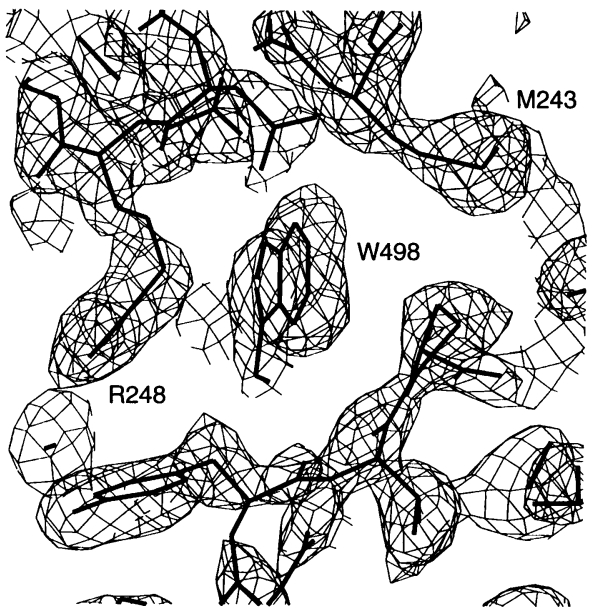

Fig. 1. Electron density at the p53-SH3 domain interface, contoured at $1.2 \sigma(25)$. The $\left(2\left|F_{\text {obs }}\right|-\right.$ $\left.\left|F_{\text {calc }}\right|\right)$ Fourier synthesis was calculated at $2.2 \AA$ resolution using phases calculated after omitting the interface residues shown, and subjecting the model to simulated annealing refinement from 3000 K. Met ${ }^{243}$ and $\operatorname{Arg}^{248}$ of p53 and Trp ${ }^{498}$ of 53BP2 are labeled.

null mice (9) do not exhibit the tumorprone phenotype of p53-null mice (10), suggesting the existence of additional mediators of $\mathrm{p} 53$ 's tumor suppressing effects.

In addition to DNA binding, several protein binding activities have been described for $\mathrm{p} 53$ (1). Among these, the binding of the 53BP2 protein, which was identified by its ability to bind the core domain of $\mathrm{p} 53$ in a yeast two-hybrid screen (11), is disrupted by alanine substitutions at several of the frequently mutated amino acids of p53 (11). To help investigate the significance of the 53BP2-p53 interaction, we have determined the $2.2 \AA$ crystal structure of the complex containing the p53 core domain and a 229-residue $\mathrm{COOH}$-terminal domain of 53BP2 (Fig. 1 and Table 1). This complex forms with a dissociation constant of $\approx 30 \mathrm{nM}$ as determined by surface plasmon resonance measurements (12).

The 53BP2 COOH-terminal domain (residues 291 to 519) contains four ankyrin repeats (13), each about 30 residues long, and a Src-homology-3 (SH3) domain (14) - two distinct structural motifs that mediate protein-protein interactions in diverse regulatory molecules. In the crystal structure of the 53BP2-p53 complex, the ankyrin repeats and the $\mathrm{SH} 3$ domain form a single structural domain as they pack extensively against each other (Fig. 2, A and B).

The p53 core domain structure in the complex is essentially identical to the crystal structures of free and DNA-bound p53 (15), consisting of a $\beta$ sandwich that serves as a scaffold for two large loops (the L2 and L3 loops) and a loop-sheet-helix motif (H2 